\title{
Recovery-focused cognitive-behavioural therapy for recent-onset bipolar disorder: randomised controlled pilot trial
}

Steven H. Jones, Gina Smith, Lee D. Mulligan, Fiona Lobban, Heather Law, Graham Dunn, Mary Welford, James Kelly, John Mulligan and Anthony P. Morrison

\section{Background}

Despite evidence for the effectiveness of structured psychological therapies for bipolar disorder no psychological interventions have been specifically designed to enhance personal recovery for individuals with recent-onset bipolar disorder.

\begin{abstract}
Aims
A pilot study to assess the feasibility and effectiveness of a new intervention, recovery-focused cognitive-behavioural therapy (CBT), designed in collaboration with individuals with recent-onset bipolar disorder intended to improve clinical and personal recovery outcomes.
\end{abstract}

\section{Method}

A single, blind randomised controlled trial compared treatment as usual (TAU) with recovery-focused CBT plus TAU $(n=67)$.

\section{Results}

Recruitment and follow-up rates within 10\% of pre-planned targets to 12-month follow-up were achieved. An average of $14.15 \mathrm{~h}$ (s.d. $=4.21$ ) of recovery-focused CBT were attended out of a potential maximum of $18 \mathrm{~h}$. Compared with TAU, recovery-focused CBT significantly improved personal recovery up to 12-month follow-up (Bipolar Recovery Questionnaire mean score 310.87, 95\% Cl 75.00-546.74 (s.e. $=120.34), P=0.010, d=0.62)$ and increased time to any mood relapse during up to 15 months follow-up $\left(\chi^{2}=7.64, P<0.006\right.$, estimated hazard ratio $(H R)=0.38$, $95 \% \mathrm{Cl} 0.18-0.78)$. Groups did not differ with respect to medication adherence.

\section{Conclusions}

Recovery-focused CBT seems promising with respect to feasibility and potential clinical effectiveness. Clinical- and cost-effectiveness now need to be reliably estimated in a definitive trial.

\section{Declaration of interest}

None.
Associative mechanisms seem to build up over repeated mood episodes in bipolar disorder, such that later episodes are both more readily triggered by psychosocial circumstances previously linked to mood changes and are also less likely to be mediated by psychosocial processes. ${ }^{1-3}$ This suggests that cognitive-behavioural interventions could be more effective if offered earlier in the course of the disorder before strong associative links to bipolar emotional states are established. Consistent with this, a post hoc analysis of data from a large trial of cognitive-behavioural therapy (CBT) for relapse prevention in bipolar disorder found a significant benefit only for those with fewer episodes. ${ }^{4}$ There is further evidence that earlier onset of bipolar disorder is linked to worse clinical outcomes, leading to calls for more timely detection and intervention. ${ }^{5,6}$ In addition to adaptations to facilitate early treatment, existing CBT interventions also need to accommodate the shift in focus from primarily symptom-reduction outcomes to include more personal-recovery outcomes, supported by both service users and UK government policy. ${ }^{7-9}$ This study is the first to evaluate the feasibility and effectiveness of an adapted CBT intervention intended to enhance personal recovery and reduce relapse for individuals within the first 5 years of bipolar disorder onset.

\section{Method}

This study was reviewed and approved by the UK NHS Ethics Committee process (REC ref: 10/H1014/60). A detailed protocol for the study has already been published. ${ }^{10}$

\section{Trial design}

This is a rater-masked pilot randomised controlled trial that compared up to $18 \mathrm{~h}$ of recovery-focused CBT for bipolar disorder offered in addition to treatment as usual (TAU) with TAU only. The trial was conducted across 11 NHS trusts in the North West of England: participants were recruited between 9 February 2011 and 19 January 2012. Individuals were randomised by an independent clinical trials unit, with minimisation on number of previous episodes $(1-6,>6)$ and current mood symptoms (depression: Hamilton Rating Scale for Depression (HRSD) ${ }^{11}$ $0-13,<13$ and mania: Bech-Rafaelsen Mania Scale (MAS) ${ }^{12}$ $0-20,>20)$, all significant predictors of therapy outcome. ${ }^{4,13,14}$ Researchers were masked to treatment condition. To maximise masking, researchers and therapists occupied different offices, researchers were not involved in randomisation and participants were reminded not to talk about treatment allocation by researchers. Fourteen breaks to masking occurred; in each case another masked researcher undertook all remaining assessments. In total, 79\% $(n=53)$ of participants had masked assessments throughout and $95 \%$ of all assessment sessions were confirmed as definitely masked. As a pilot study, the sample size was selected to determine feasibility and acceptability and to provide indicative information on potential clinical effectiveness for a future definitive trial (for which an attention control condition would be considered along with longer follow-up).

\section{Recruitment}

Community mental health teams, out-patient clinics, general practice surgeries, primary care mental health teams and voluntary services were approached to identify potential participants. Care coordinators, research nurses and research development officers were approached in order to contact potential participants in the first instance. The study was also 
advertised in local media and posters and leaflets distributed in both National Health Service (NHS) and non-NHS sites to maximise participant access. Written informed consent was obtained from all participants.

\section{Inclusion/exclusion criteria}

All participants met the following inclusion criteria: (a) DSM-IV ${ }^{15}$ diagnosis of primary bipolar disorder with onset in past 5 years, assessed using the Structural Clinical Interview for DSM-IV (SCID) $;^{16}$ (b) sufficient understanding of written and spoken English in order to provide consent, engage with interviews and use the intervention; and (c) aged between 18 and 65 years. Exclusion criteria included: manic, hypomanic, depressed or mixed episode currently or in the past 4 weeks, so that individuals were able to engage with a therapy focused on personal recovery outcomes rather than acute symptom management.

\section{Outcome measures}

Feasibility and acceptability of delivering the recovery-focused CBT intervention to individuals with recent-onset bipolar disorder (onset in past 5 years) was measured by the following factors: levels of recruitment into the trial; retention of participants in both arms of the study and adherence to and completion of the intervention. Participants were followed up for up to 15 months from initial randomisation with telephone assessments every 3 months to evaluate bipolar relapse and observer-rated mood (SCID-LIFE, ${ }^{17}$ $\mathrm{HRSD}^{11}$ and MAS ${ }^{12}$ ). Self-reported recovery (Bipolar Recovery Questionnaire, $\mathrm{BRQ}^{18}$ ) and additional clinical measures were assessed at baseline and then at 6 and 12 months (see below for detail on the assessments used). It was originally intended to collect both self-report and observer-reported data on all participants for up to 18 months' follow-up. However, it proved impossible to achieve this final intended assessment within the trial funding period because of delays with staff recruitment and trust research and development approvals and limited therapist capacity. Recovery data (self-report) is reported for up to 12 months and relapse/observer-rated symptom data for up to 15 months (12 months for 17 participants whose 15 -month assessment point fell after the study period).

\section{Primary clinical outcomes}

Primary clinical outcomes (as pre-specified in the published protocol ${ }^{10}$ ) assessed the effect of recovery-focused CBT on selfreported recovery measured by the BRQ (33-item version used, correlates 0.997 with the published 36 -item version ${ }^{18}$ ), time to bipolar relapse measured by the SCID-LIFE and mood symptoms as measured by the HRSD and MAS. The BRQ items were scored on a $0-100$ scale (higher score, sum of items, indicates higher personal recovery).

\section{Secondary clinical outcomes}

Secondary outcomes evaluated were the impact of recoveryfocused CBT on the following: (a) quality of life (Brief Quality of Life in Bipolar Disorder Questionnaire, QoL.BD $)^{19}$ and social functioning (Personal and Social Functioning Scale; PSP); ${ }^{20}$ (b) self-reported mood symptoms as measured by the Beck Depression Inventory (BDI-II $)^{21}$ and Internal States Scale (ISS; activation (ISS-A) and wellbeing (ISS-W) subscales); ${ }^{22}$ (c) medication adherence (days missed for prescribed medication self-report) measured by the Stephenson Medical Adherence Questionnaire (MEDAD). ${ }^{23}$

\section{Therapeutic alliance and adherence to treatment protocol}

Therapy engagement was assessed by attendance rates and Working Alliance Inventory (short-form, therapist and client versions; WAI-S) completed at sessions 4,8 , and 17 to sample from early, middle and later phases of therapy. ${ }^{24}$ Treatment fidelity was assessed by both the Cognitive Therapy Scale Revised version $(\mathrm{CTS}-\mathrm{R})^{25}$ and the Recovery Fidelity Scale, specifically designed for the current study (details available from the authors on request), to indicate adherence to the therapy's recovery focus, again sampled across early, middle and late phases of therapy.

\section{Individual recovery-focused CBT intervention}

The intervention was informed by key components of effective CBT interventions ${ }^{26}$ and findings from a case series study of CBT for recently diagnosed bipolar disorder. ${ }^{27}$ Key features include flexible engagement, allowing time for full consideration of the meaning of diagnosis to the client, coping skills reviews around subsyndromal exacerbations as well as prior episodes and considering the role of appraisals of fluctuations in affect. Therapy manual development was also informed by qualitative interviews with individuals about their experiences of recovery in bipolar disorder and focus groups with individuals with bipolar disorder to consult on the draft content of therapy, format and supporting materials for the recovery-focused CBT intervention. The intervention was delivered by mental health professionals trained to British Association for Behavioural and Cognitive Psychotherapies accreditation level in CBT or equivalent. Duration of therapy was up to $18 \mathrm{~h}$ delivered over approximately 6 months at clients' homes or mental health facilities, according to personal preference. Initial sessions were weekly, with later sessions fortnightly, and typically lasted $45-60 \mathrm{~min}$.

The following elements are contained within the recoveryfocused CBT manual and reflect elements typically addressed in the course of therapy, although the relative emphasis on each element is informed by the client's formulation: introducing the recovery approach to clients; collection of information about current and historical mood and functioning; meaning and relevance of diagnosis; identification of recovery-informed therapy goals; initial formulation of relationships between mood experiences and progress towards recovery goals; identification and application of CBT techniques to address and facilitate positive coping; consideration of wider functioning issues in relation to recovery; development and completion of recovery plan; sharing lessons from therapy with key stakeholders.

The therapy approach differs from standard CBT for bipolar disorder in the following ways: explicit focus on eliciting clientfocused goals rather than presuming a target of relapse prevention; formulation-driven idiosyncratic approach rather than applying a very similar model of bipolar experience across clients; freedom to work within whatever model the client brings; openness to addressing functioning and comorbidity issues as well as mood problems; emphasis on supporting clients to move away from self-critical and/or stigmatising language, especially around diagnosis and behaviour in acute episodes.

In both groups, TAU was characterised by routine medication (mood stabilisers, antipsychotics and antidepressants). Over twothirds of participants received their medical care from secondary care services, mainly through a combination of maintenance appointments from their responsible clinician and support from a community mental health team (CMHT). A small number of participants were being treated by a psychiatrist only without regular CMHT contact. The remainder of participants reported attending primary care services. 


\section{Therapists}

Recovery-focused CBT was delivered by four therapists, all of whom were qualified clinical psychologists. One therapist saw the majority of clients (G.S. $n=21$ ), the other therapists saw two or five clients each (M.W. $n=2$; J.K. and J.M. $n=5$ ). All therapists received training in the use of the recovery-focused CBT manual and attended weekly therapy supervision.

\section{Statistical analysis}

Analyses were carried out using Stata version 11 for Windows and were pre-specified. ${ }^{10}$ All therapy effects were estimated using a random-effects (random intercepts) model, assuming that the effects were the same for each follow-up time (having first checked that there was no significant therapy by follow-up time interaction). The baseline value of the relevant outcome measure was used as a covariate. The intention-to-treat principle was followed throughout. If there had been a significant number of participants not attending their allocated sessions, this would have been supplemented by estimation of the therapy effects in those participants who actually receive the intervention via estimation of the Complier-Average Causal effect (CACE). ${ }^{28,29}$ However, this proved to be unnecessary. Time to relapse was assessed by survival analysis (differences between groups being evaluated using the logrank test; hazard ratios (HRs) were estimated using a Cox proportional hazards model). Missing data were assumed to be missing at random (ignorable) and automatically allowed for in fitting the random-effects or analysis of covariance models. ${ }^{30}$

\section{Results}

\section{Feasibility outcomes}

Feasibility outcomes for this study were assessed by recruitment and retention rates in both arms and participation in the recovery-focused CBT for those in the treatment arm. Participant flow is indicated in Fig. 1. Of 125 screened for eligibility, 67 individuals were randomised to recovery-focused CBT or TAU. Of those not randomised, the majority declined the opportunity to participate or did not respond to initial contact $(n=35,19$ did not respond to initial contact, 4 refused to participate in the screening for the study, 12 could not commit to the full study period including follow-up assessments). The remainder did not meet study criteria because of a duration of illness greater than 5 years $(n=14)$, currently in an episode $(n=6)$, older than 65 years $(n=2)$ or already participating in another intervention study $(n=1)$.

Of the 67 participants randomised, $78 \%(n=52)$ were retained to end of therapy follow-up at 6 months, $76 \%(n=51)$ to 9 -month follow-up and $67 \%(n=45)$ at 12-month follow-up. The 15-month follow-up period was tapered as study funding terminated before this assessment became due for 17 participants. Of those eligible, $62 \%(n=31)$ completed 15 -month follow-up.

Of the 33 participants allocated to recovery-focused CBT, 32 attended at least six sessions (mean 14.15 h, s.d. $=4.21$ ). Only four clients expressed a preference not to receive therapy at home because of either family conflicts or practical convenience (one was seen in a community mental health team, two at their GP practice and one at their place of work). Working alliance ratings were obtained from therapists for 69 sessions and from clients for 51 sessions (47 paired ratings). Alliance as rated by therapists was 56.55 (s.d. $=4.94)$ at session $4 ; 58.84($ s.d. $=4.76)$ at session 8 ; and 61.76 (s.d. $=4.84$ ) at session 17. At each stage of therapy clients' ratings were higher than those of therapists: 64.72 (s.d.=7.01); 66.35 (s.d.=7.66); and 71 (s.d.=5.51), respectively. Alliance

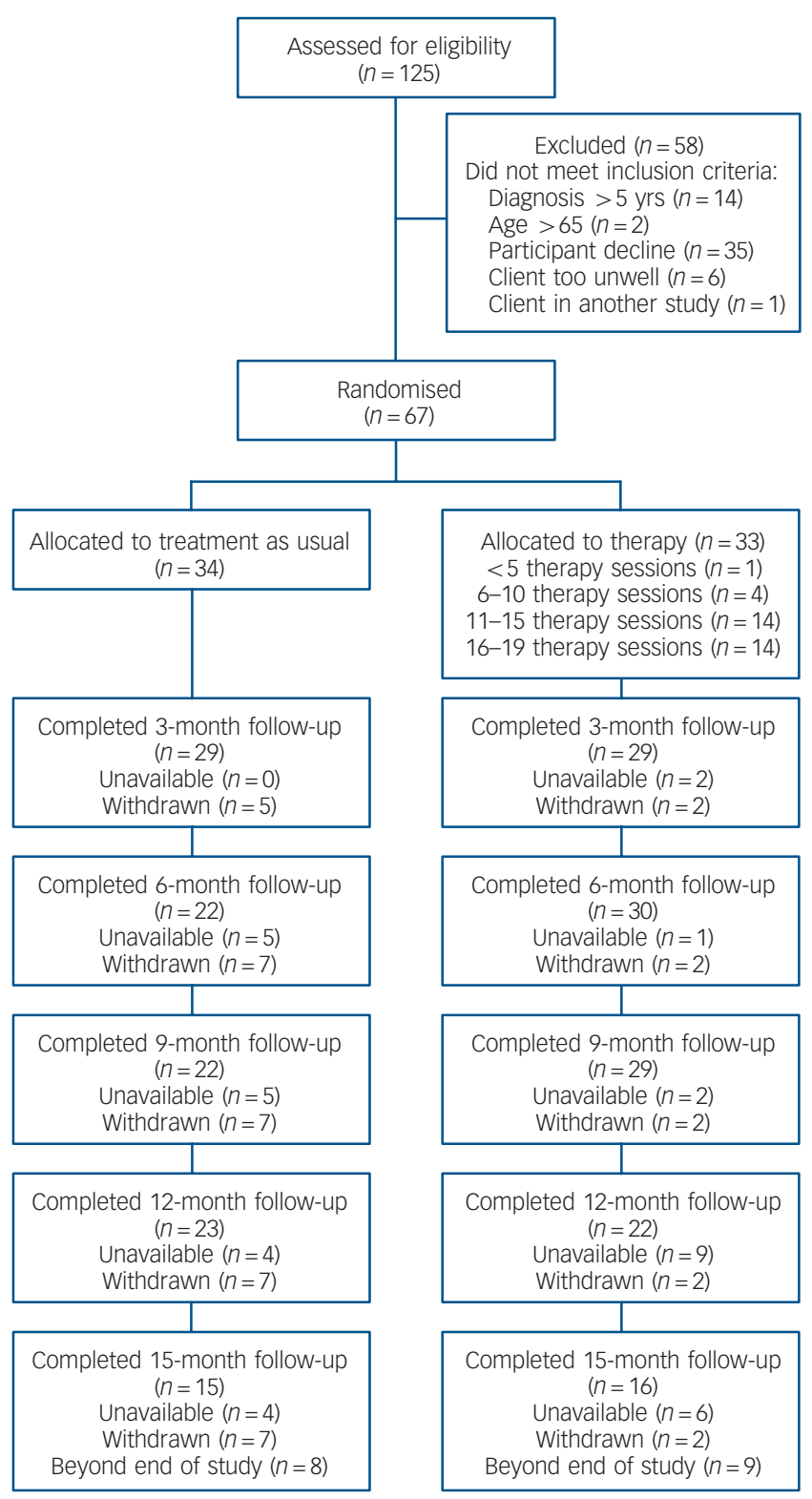

Fig. 1 CONSORT diagram.

ratings are comparable with those observed for psychological therapy in mild and complex patient groups. ${ }^{31,32}$ Therapist protocol adherence was independently assessed for 33 randomly selected session tapes. Adherence to the therapy protocol was 91\% (Recovery Fidelity Scale), whereas the mean score for CTS-R was 35.4 (s.d.=7.9), which is above the established threshold criteria for competence. ${ }^{33}$ Recovery Fidelity Scale and CTS-R scores were moderately but significantly correlated $(r=0.467, P<0.006)$.

\section{Participant characteristics}

Participants were on average $<40$ years old and predominantly female $(70 \%, n=47)$ in both groups, and received their clinical diagnosis of bipolar disorder within the past 2-3 years (Table 1). A small proportion of participants had an early age at onset of bipolar disorder ( $<18$ years, $n=6$ TAU group; $n=7$ recoveryfocused CBT group). The majority of participants $(79 \%, n=53)$ had a diagnosis of bipolar I disorder (the remainder had bipolar II disorder) and subclinical scores on observer measures of depression and mania. Participants in both groups were receiving 


\begin{tabular}{|c|c|c|c|c|c|}
\hline & $\begin{array}{l}\text { Treatment as usual group } \\
\qquad(n=34)\end{array}$ & $\begin{array}{l}\text { Therapy group } \\
\qquad(n=33)\end{array}$ & $t$ & $\chi^{2}$ & $P$ \\
\hline Age, mean (s.d.) & $39.9(10.4)$ & $38.3(12.8)$ & 0.58 & & $<0.58$ \\
\hline Gender, female: $n$ (\%) & $22(64.7)$ & $25(75.8)$ & & 0.98 & $<0.34$ \\
\hline Age of bipolar diagnosis, mean (s.d.) & $37.4(10.2)$ & $35.9(12.7)$ & -0.38 & & $<0.70$ \\
\hline \multicolumn{6}{|l|}{ Diagnosis } \\
\hline Bipolar I disorder, $n$ (\%) & $29(85.3)$ & $24(72.7)$ & & 1.60 & $<0.21$ \\
\hline Bipolar II disorder, $n$ (\%) & $5(14.7)$ & $9(27.3)$ & & & \\
\hline \multicolumn{6}{|l|}{ Number of previous episodes, $n$ (\%) } \\
\hline $1-6$ & $14(41.2)$ & $14(42.4)$ & & 0.01 & 0.92 \\
\hline$>6$ & $20(58.8)$ & $19(57.6)$ & & & \\
\hline \multicolumn{6}{|l|}{ Depression (HRSD), $n$ (\%) } \\
\hline $0-13$ & $26(76.5)$ & $27(81.8)$ & & 0.29 & $<0.59$ \\
\hline$>13$ & $8(23.5)$ & $6(18.2)$ & & & \\
\hline \multicolumn{6}{|l|}{ Mania (MAS), $n(\%)$} \\
\hline $0-20$ & $34(100)$ & $33(100)$ & & & \\
\hline$>20$ & 0 & 0 & & & \\
\hline \multicolumn{6}{|l|}{ Medication at baseline, $n$ (\%) } \\
\hline Antidepressants & $17(50)$ & $14(42)$ & & 0.39 & $<0.53$ \\
\hline Mood stabilisers & $22(65)$ & $14(42)$ & & 3.34 & $<0.07$ \\
\hline Antipsychotics & $21(62)$ & $20(61)$ & & 0.01 & $<0.92$ \\
\hline \multicolumn{6}{|l|}{ Care team, $n(\%)$} \\
\hline Community mental health team & $24(71)$ & $19(58)$ & & 1.24 & $<0.53$ \\
\hline Psychiatrist & $3(9)$ & $4(12)$ & & & \\
\hline General practitioner & $7(21)$ & $10(30)$ & & & \\
\hline
\end{tabular}

a mixture of antidepressants, mood stabilisers and antipsychotics. Care team arrangements were similar, with the majority $(75 \%$, $n=50$ ) of both groups receiving care from a community mental health team or primarily from a psychiatrist. A minority $(25 \%$, $n=17$ ) of participants in both groups were under the care of their GP. There were no significant differences between groups on any of these variables. Online Table DS1 provides supplementary demographic information concerning marital status, ethnicity, schooling, employment, and admissions to hospital. The two groups did not differ on any of these variables.

\section{Baseline scores}

Both groups had low levels of depression and mania at baseline, consistent with entry criterion of being out of a current mood episode. Scores on the BRQ and QoL.BD indicate moderate levels

\begin{tabular}{|c|c|c|c|c|c|c|c|c|}
\hline & \multicolumn{3}{|c|}{ Treatment as usual group } & \multicolumn{3}{|c|}{ Recovery-focused CBT group } & \multicolumn{2}{|c|}{ Test statistics } \\
\hline & $n$ & Mean & s.d. & $n$ & Mean & s.d. & $t$ & $P$ \\
\hline \multicolumn{9}{|c|}{ Bipolar Recovery Questionnaire } \\
\hline Baseline & 33 & 1934.57 & 543.85 & 33 & 1797.39 & 454.80 & \multirow[t]{3}{*}{1.115} & \multirow[t]{3}{*}{$<0.27$} \\
\hline 6-month follow-up & 21 & 2082.57 & 518.58 & 26 & 2378.92 & 578.26 & & \\
\hline 12-month follow-up & 15 & 2193.40 & 357.74 & 22 & 2351.41 & 462.02 & & \\
\hline \multicolumn{9}{|c|}{ Quality of Life in Bipolar Disorder Scale } \\
\hline Baseline & 33 & 36.73 & 11.59 & 33 & 35.91 & 8.76 & \multirow[t]{3}{*}{0.32} & \multirow[t]{3}{*}{$<0.75$} \\
\hline 6-month follow-up & 21 & 38.14 & 14.27 & 26 & 42.30 & 9.03 & & \\
\hline 12-month follow-up & 15 & 39.67 & 7.60 & 22 & 42.27 & 7.34 & & \\
\hline \multicolumn{9}{|c|}{ Personal and Social Performance Scale } \\
\hline Baseline & 33 & 72.45 & 16.80 & 33 & 67.97 & 18.05 & \multirow[t]{3}{*}{0.58} & \multirow[t]{3}{*}{$<0.30$} \\
\hline 6-month follow-up & 18 & 68.50 & 18.67 & 22 & 75.73 & 19.52 & & \\
\hline 12-month follow-up & 16 & 77.13 & 15.78 & 18 & 78.44 & 18.10 & & \\
\hline \multicolumn{9}{|c|}{ Internal States Scale - Activation } \\
\hline Baseline & 33 & 117.18 & 95.37 & 33 & 115.91 & 90.98 & \multirow[t]{3}{*}{0.055} & \multirow[t]{3}{*}{$<0.96$} \\
\hline 6-month follow-up & 19 & 100.32 & 98.37 & 24 & 81.96 & 77.89 & & \\
\hline 12-month follow-up & 15 & 93.26 & 76.87 & 22 & 93.86 & 101.93 & & \\
\hline \multicolumn{9}{|c|}{ Internal States Scale - Wellbeing } \\
\hline Baseline & 33 & 131.55 & 86.16 & 33 & 132.09 & 73.47 & \multirow[t]{3}{*}{-0.027} & \multirow[t]{3}{*}{$<0.98$} \\
\hline 6-month follow-up & 19 & 157.00 & 62.63 & 24 & 165.88 & 69.25 & & \\
\hline 12-month follow-up & 15 & 153.60 & 78.90 & 22 & 162.14 & 78.00 & & \\
\hline \multicolumn{9}{|c|}{ Beck Depression Inventory } \\
\hline Baseline & 33 & 19.00 & 14.85 & 33 & 19.39 & 13.23 & \multirow[t]{3}{*}{-0.113} & \multirow[t]{3}{*}{$<0.91$} \\
\hline 6-month follow-up & 20 & 13.60 & 13.00 & 26 & 13.58 & 14.91 & & \\
\hline 12-month follow-up & 15 & 14.87 & 14.20 & 22 & 14.41 & 21.98 & & \\
\hline
\end{tabular}


of personal recovery and quality of life at baseline (Table 2). There were no significant differences between groups on any of these variables.

\section{Primary clinical outcomes}

The BRQ score was higher in the recovery-focused CBT group at follow-up than the TAU group $(310.87,95 \%$ CI $75.00-546.74$ (s.e. $=120.34), P=0.010, d=0.62)$ with no interaction between this effect and follow-up assessment point (6 or 12 months), indicating greater improvement in recovery after therapy, sustained at follow-up. As Table 2 indicates, not all participants completed self-report questionnaires at assessment points (BRQ data $98 \%(n=66)$ of potential sample at baseline, $90 \%(n=47)$ at 6 months, and $82 \%(n=37)$ at 12 months $)$.

Follow-up relapse data were available for 58 participants $(29$ from each of the two treatment groups). Participants were followed up for a period of up to 15 months during which time 32 experienced a relapse of either depression or mania (20 TAU v. 12 recovery-focused CBT). Kaplan-Meier estimates of time to first recurrence of either type are presented in Fig. 2. Median survival times for the TAU and recovery-focused CBT groups were 18 (95\% CI 8-40) and 56 (95\% CI 28-8) weeks, respectively. The difference in time to recurrence was statistically significant $\left(\chi^{2}=7.64, P<0.006\right.$, estimated $\mathrm{HR}=0.38,95 \%$ CI $\left.0.18-0.78\right)$.

During follow-up, 30 patients experienced a depressive relapse (19 TAU v. 11 recovery-focused CBT). Kaplan-Meier estimates of time to first depressive recurrence are presented in Fig. 3. Median survival times for the TAU and recovery-focused CBT groups were 18 (95\% CI 8-47) and 60 (95\% CI 29-8) weeks, respectively. The difference in time to recurrence rates was statistically significant $\left(\chi^{2}=7.63, P<0.006\right.$, estimated HR $=0.37,95 \%$ CI $\left.0.18-0.77\right)$.

Manic relapses were less common, occurring in 13 participants (10 TAU v. 3 recovery-focused CBT). Times to first manic recurrence are presented in Fig. 4. Median survival times for the TAU and recovery-focused CBT groups were 33 (95\% CI 23-58) and $60(95 \%$ CI 53-8) weeks, respectively. This difference in time to recurrence was statistically significant $\left(\chi^{2}=6.77\right.$, $P<0.009$, estimated HR $=0.38,95 \%$ CI $0.19-0.79)$.

Comparisons were also made for average mood symptoms (HRSD and MAS) (Figs 5 and 6). There was no significant impact of recovery-focused CBT compared with TAU on depressive (HRSD, $-0.98,95 \%$ CI -3.66 to 1.71 (s.e. $=1.37), d=-0.17$ ) or manic symptoms (MAS, $-0.66,95 \%$ CI -1.69 to 0.37 (s.e. $=0.53), d=-0.19$ ) across the follow-up period although the direction of effect favoured recovery-focused CBT in both cases: both depressive and manic symptom scores were low throughout the study period.

\section{Secondary clinical outcomes}

Table 2 provides summary data for quality of life (QoL.BD), social functioning (PSP) and self-reported mood symptoms (BDI and ISS). For QoL.BD and PSP there were non-significant trends towards greater improvements with recovery-focused CBT at follow-up compared with TAU (QoL.BD 4.83, 95\% CI -0.07 to 9.74 (s.e. $=2.50$ ), $P=0.054, d=0.47$; PSP $6.12,95 \% \mathrm{CI}-2.22$ to 14.46 (s.e. $=4.25$ ) $, P=0.151, d=0.35$ ). There was no interaction between either effect and follow-up points (6 or 12 months).

There was no evidence for a significant differential treatment effect on self-reported mood symptoms, although in all cases the direction of effect favoured recovery-focused CBT (for ISS-A and BDI lower score, and for ISS-W higher score, indicated improvement: ISS-A $-2.79, \quad 95 \%$ CI -37.86 to 32.27 (s.e. $=17.98), P=0.88, d=-0.03$; ISS-W $8.09,95 \%$ CI -25.32

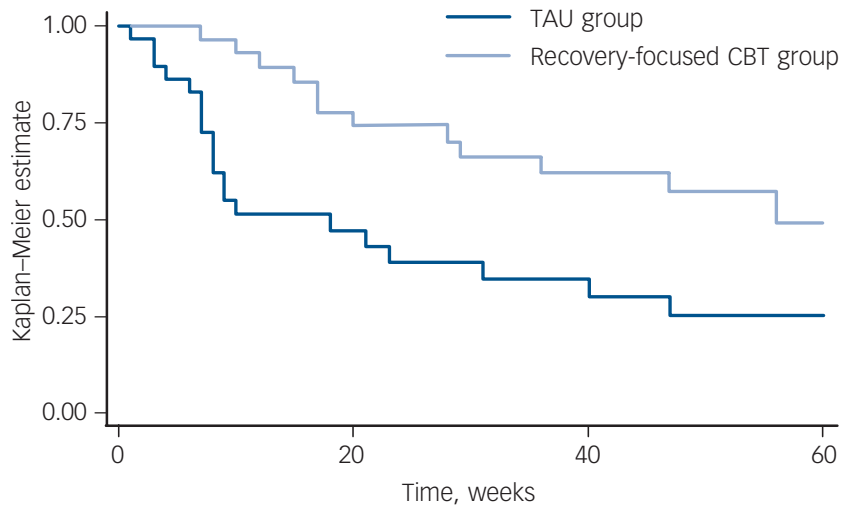

Fig. 2 Kaplan-Meier estimates of time to first depressive or manic recurrence over up to 60 weeks follow-up.

TAU, treatment as usual; CBT, cognitive-behavioural therapy.

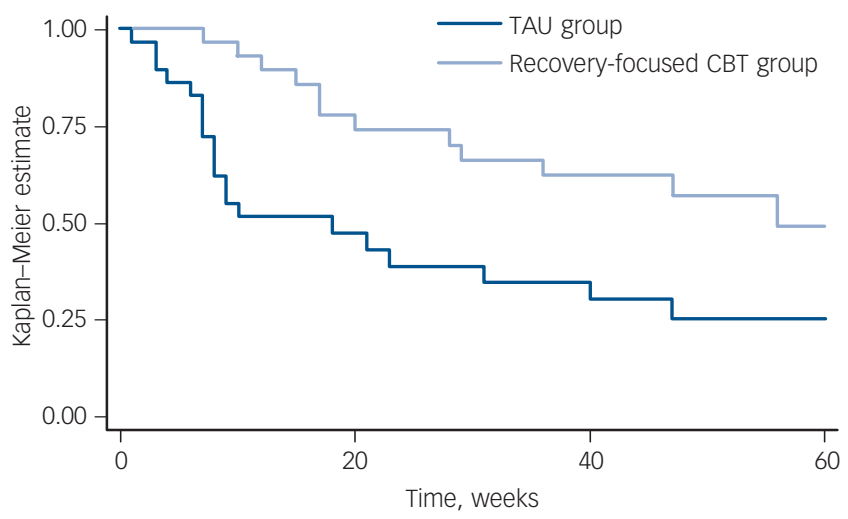

Fig. 3 Kaplan-Meier estimates of time for depressive recurrence over up to 60 weeks follow-up.

TAU, treatment as usual; CBT, cognitive-behavioural therapy.

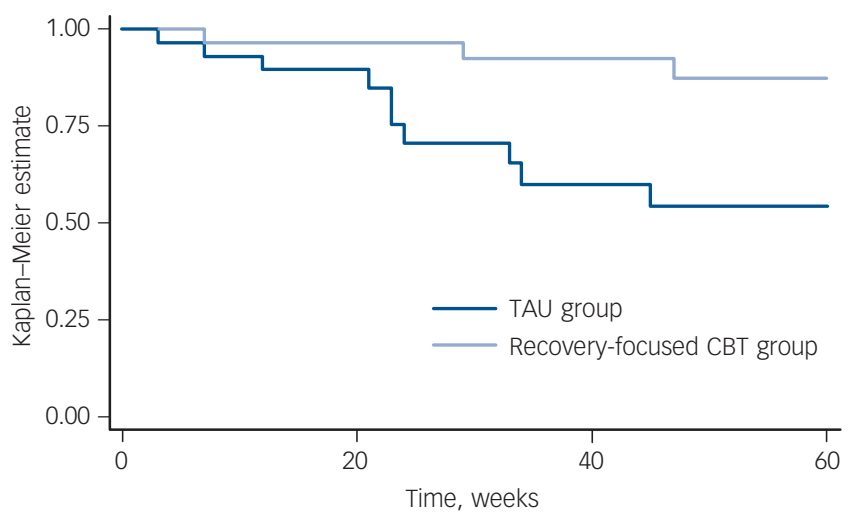

Fig. 4 Kaplan-Meier estimates for time to first manic recurrence over up to 60 weeks follow-up.

TAU, treatment as usual; CBT, cognitive-behavioural therapy.

to 41.51 (s.e. $=17.04), P=0.635, d=0.10$; BDI $-0.88,95 \% \mathrm{CI}$ -7.59 to 5.84 (s.e. $=3.43$ ), $P=0.798, d=-0.05$ ).

Medication adherence (number of days medication missed in the preceding 4 weeks) was compared for antipsychotics, mood stabilisers and antidepressants (in participants for whom these were prescribed) at baseline, 6 months and 12 months. Selfreported adherence was high across assessments (antidepressants: 


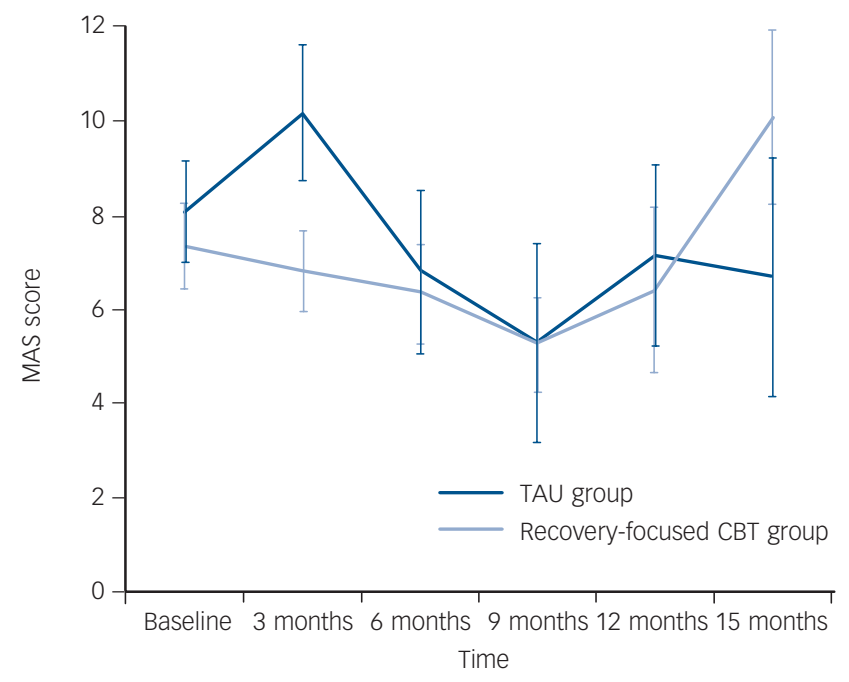

Fig. 5 Observer-rated depression scores on the Hamilton Rating Scale for Depression (HRSD).

$\mathrm{TAU}$, treatment as usual; CBT, cognitive-behavioural therapy.

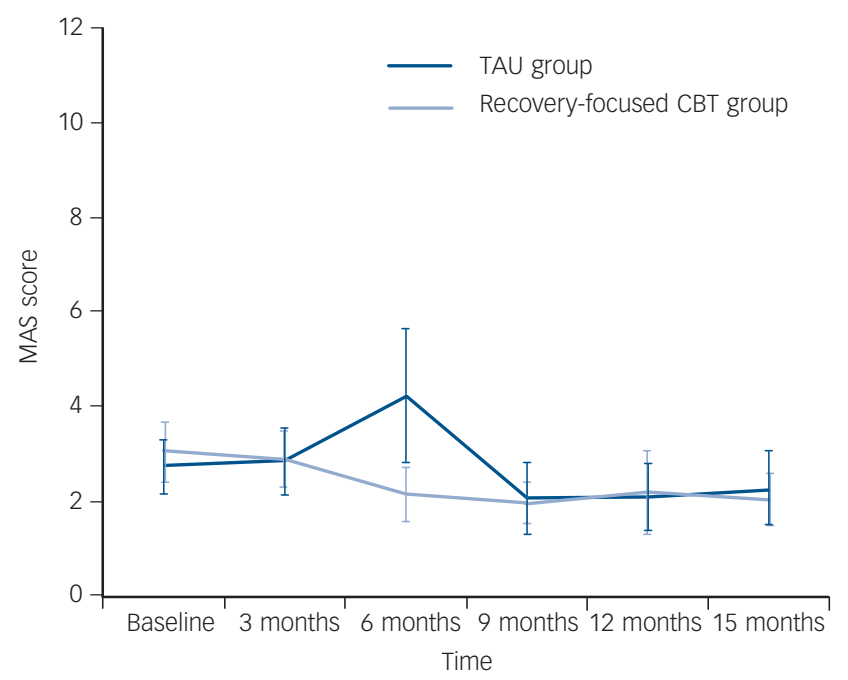

Fig. 6 Observer-rated mania scores on the Bech-Refaelsen Mania Scale (MAS)

TAU, treatment as usual; CBT, cognitive-behavioural therapy.

means range 0-6 days missed; mood stabilisers: means range $0.2-$ 3.8 days missed; antipsychotics: means range $0.2-4$ days missed). No significant differences were found between recovery-focused CBT and TAU at any assessment point.

\section{Discussion}

This is the first study to evaluate the impact of recovery-focused CBT on recent-onset bipolar disorder. Recovery-focused CBT was developed in partnership with individuals with lived experience of bipolar, consistent with Mental Health Research Network good practice guidelines. ${ }^{34}$ The high level of engagement of individuals with personal experience of bipolar disorder is consistent with the aims of recovery approaches ${ }^{7-9}$ to be empowering, individualised and grounded in the individual's own priorities and needs. Recovery-focused CBT differs from standard CBT in having less of a focus on relapse prevention and symptom reduction. ${ }^{4,35}$ There is also a strong emphasis on formulation, so that the client's route through therapy is different depending on their needs, giving the therapist freedom to work with whatever model the client brings but in the context of offering evidence-informed approaches. Therapists are also encouraged to focus on issues around functioning and comorbidity as well as mood problems, should these be prioritised by the client.

\section{Feasibility and effectiveness of recovery-focused CBT}

Findings provide preliminary evidence in support of both feasibility and effectiveness of recovery-focused CBT for people with recently diagnosed bipolar disorder. With respect to feasibility, it proved possible to recruit participants to within $93 \%$ of the target figure of 72 within 12 months with a single whole-time equivalent research assistant. Of the potential participants screened, a key reason for the $7 \%$ shortfall was participants becoming unwell between initial screening and baseline. With more time and resources, the recruitment target would have been fully met as there was substantial interest in recovery-focused CBT among service users and clinicians. In total, $54 \%$ of potential participants screened met eligibility criteria and were randomised: this compares well with the Scott et al's ${ }^{4}$ and Lam et als ${ }^{35}$ trials, for which the figures were $17 \%$ and $48 \%$, respectively. Retention to follow-up assessments was balanced across both arms of the study and within $10 \%$ of the $75 \%$ target to 12 -month follow-up. Scott et al reported retention rates of $78 \%$ to final follow-up in their trial, ${ }^{4}$ whereas Lam et al's data is unclear on drop-out rates post therapy. ${ }^{35}$

Participants in the therapy arm engaged with recovery-focused CBT, attending 14 therapy sessions on average with 32 clients attending $\geqslant 6$ sessions, which is comparable with previous studies. ${ }^{4,35}$ Over $80 \%$ of clients preferred to receive therapy in their home. Therapists reported that clients appreciated the flexibility of home-based therapy and felt that it enhanced engagement compared with an office-based approach. The main challenges experienced in delivering therapy in this way were that sessions tended to be longer and that timing of the discussion of sensitive issues needed to negotiated flexibly (such as when there was no other family member in the house). Both client and therapist ratings of working alliance were consistent with clients experiencing the therapy as worthwhile. Therapy adherence ratings indicated that the therapy delivered was consistent with CBT good practice principles and also with the more flexible recovery focus of recovery-focused CBT. As the CTS-R and Recovery Fidelity Scales were only moderately correlated, the importance of developing tailored measures where the trial intervention has the more flexible structure inherent in a formulation-driven intervention is supported. Previous studies of established bipolar disorder have not reported working alliance data or recovery adherence but reported similar ratings for therapist CBT competency. ${ }^{4}$

In terms of primary clinical outcomes, recovery-focused CBT significantly improved personal ratings of recovery at both 6- and 12-month follow-up assessments, compared with TAU. The treatment effect is moderate-large (baseline to end therapy: $30 \%$ improvement in the recovery-focused CBT group compared with $8 \%$ in the TAU group; average item score moved from neutral to agree in recovery-focused CBT and remained neutral in TAU) and suggests moderate change across the BRQ domains of selfmanagement of health, access to personally valued activities, seeing recovery as a lifelong process and understanding mood experiences. Future research should aim to determine the minimal clinically important difference for BRQ to inform a future 
definitive trial. Recovery-focused CBT also significantly increased time to any relapse and time to both depressive and manic relapses. Median time to any relapse was increased by 38 weeks, time to depressive relapse by 42 weeks and time to manic relapse by 27 weeks, compared with TAU. By contrast, recovery-focused CBT had no significant impact on observer ratings of residual symptoms of depression or mania. Both HRSD and MAS scores were low throughout the study - for the HRSD, the scores ranged from mild depression to no depression across follow-ups, whereas for the MAS, mania scores indicated absence of mania throughout on average.

For secondary outcomes, the clinical impact of recoveryfocused CBT seemed to be marginally stronger on functional than on symptomatic measures. Thus, quality of life and social functioning were numerically improved in recovery-focused CBT compared with TAU, with moderate effect sizes (baseline to end therapy: QoL.BD average item score moved from disagree/neutral to neutral/agree for recovery-focused CBT and neutral at both points for TAU; PSP moved from manifest problems to mild in recovery-focused CBT and from mild to manifest in TAU), but differences were not statistically significant. There were no group differences on self-reported mood. Both groups scored in the no mania range on ISS-A throughout the study, whereas for ISS-W both groups scored as mildly depressed at baseline, but outside the clinical range at both follow-up points. The BDI scores indicated mild-minimal depression throughout the study period.

Medication adherence was high across baseline and follow-up assessments, consistent with previous reports for CBT for bipolar disorder. ${ }^{35}$ There was no evidence for a significant difference between groups in number of days on which medication was missed at any assessment points so it seems unlikely that the impact on recovery and relapse observed here are related to medication adherence changes. There was a non-significant trend towards fewer participants in the recovery-focused CBT group receiving mood stabilisers at baseline. This would if anything exert a conservative effect on the reported findings (as recovery-focused CBT group experienced fewer relapses).

\section{Comparison with findings from other studies}

No other CBT studies have specifically explored recovery outcomes in bipolar disorder, so direct comparisons for this variable are not possible. Fowler $e t a l^{36}$ reported on the effect of an adapted CBT intervention to enhance social recovery in psychosis, which included individuals with affective (including bipolar disorder) and non-affective psychosis. In contrast to the present study, Fowler et $a l^{36}$ reported that improvements in social recovery (amount of time engaged in constructive/structured activity) were limited to those with non-affective psychosis. However, the authors argued that their subsample of participants with affective psychosis responded strongly to TAU (active case management), which might have masked potential benefits of their recovery intervention.

Previous studies of CBT in bipolar disorder have varied in their impact on time to relapse. Lam et al reported a significant effect over 12 months, ${ }^{35}$ whereas Ball et al reported a trend ${ }^{37}$ (for depressive relapse), both favouring CBT in increasing survival time. By contrast, neither Scott et $a l^{4}$ nor Meyer \& Hautzinger ${ }^{38}$ identified any benefit of CBT for survival times to relapse. Zaretsky et $a l^{39}$ compared CBT with a brief individual psychoeducation intervention and reported a significant benefit for the CBT group for number of days depressed but no significant impact on relapse rate. A post hoc finding from Scott et al was that more previous episodes was associated with worse outcomes, leading to a suggestion that earlier intervention might be more appropriate. Consistent with this, our trial of recoveryfocused CBT with individuals within 5 years of diagnosis found strong effects on relapse at 15 months $(\mathrm{HR}=0.38$, relapse: $41 \%$ recovery-focused CBT v. 69\% TAU), consistent with 12-month follow-up findings reported by Lam et al ${ }^{35}(\mathrm{HR}=0.40$, relapse: $43.8 \%$ CBT v. $75 \%$ TAU) and higher than both Scott et als ${ }^{4}$ findings over 18 months ( $\mathrm{HR}=1.05$, relapse: $53 \%$ CBT v. $51 \%$ TAU) and a recent 12-month study of enhanced-relapse prevention ${ }^{40}$ (HR $=0.79$, relapse: $46 \%$ enhanced-relapse prevention v. $60 \%$ TAU). It is of interest that this was despite recovery-focused CBT not having a primarily therapeutic focus on relapse prevention alone. This impact on relapse was not associated with change in residual mood symptoms (these were consistently below clinical thresholds for HRSD and MAS throughout the trial). A possible mechanism for improved relapse outcomes is through improved personal recovery. As BRQ includes self-management of health and understanding of mood experiences, these elements may play a role in relapse prevention. However, the current study was not designed to test this proposal so further research is required to address this specific question. Of the CBT studies referred to above, only Lam et $a l^{35}$ reported benefits in social functioning following therapy. Zaretksy et $a b^{39}$ Meyer \& Hautzinger $^{38}$ and Ball et $a l^{37}$ reported no differences between groups and Scott did not report outcomes with respect to social adjustment or quality of life, although this data was collected.

There are three key areas of innovation in our study: (a) taking a formulation-driven approach to therapy for bipolar disorder; (b) focusing on personal recovery outcomes; and (c) targeting recent-onset bipolar disorder. The results indicate that this flexible approach has an impact not only on personal recovery but also on bipolar relapse; however it is unclear which of the innovative aspects may be contributing to the encouraging outcomes that were observed. A definitive trial is required to formally evaluate both the clinical efficacy and cost-effectiveness of recovery-focused CBT and to understand more fully the mechanisms that underpin the apparent benefits of this therapy.

\section{Limitations}

There are weaknesses in this study that would need to be addressed in a definitive trial. The sample size is relatively small, the study was conducted in the North West of England only and confidence intervals for effect estimates are quite large. It therefore remains to be seen how well the effects reported are replicated in a large sample. We did not correct for multiple comparisons (for example, using Bonferroni's correction), which may increase the risk of type I error. Additional issues are, first, although the current study indicates benefits for CBT over TAU alone, we do not yet have evidence for whether recovery-focused CBT has any advantage over other forms of structured treatment with an attention control to rule out non-specific effects as a cause for the observed effects. Second, it was only possible to follow up a proportion of the sample for 15 months. Longer follow-ups would be helpful to indicate more definitively the persistence of the gains reported here. Third, the cost-effectiveness of recovery-focused CBT was not assessed. Although the impact on recovery and time to recurrence suggest there may be cost savings associated with this therapy, this needs to be empirically tested. However, and perhaps most importantly, our trial had relatively low statistical power. Given the trend observed in trials of specific psychological therapies such as CBT towards reduced effect sizes with improved study quality (such as through adequate statistical power and a control condition that is more active than TAU), it is possible that our effect sizes are inflated. Therefore, an adequately powered, definitive, randomised controlled trial is required. 


\section{Clinical implications}

Recovery-focused CBT is the first therapy for bipolar disorder that explicitly aims to enhance personal recovery outcomes as supported by both service users and UK government policy. ${ }^{7-9}$ The current study indicates that recovery-focused CBT is feasible and can improve personal recovery and time to relapse. This study highlights the potential benefits of taking a formulation-based approach to bipolar disorder, in which a range of evidence-informed techniques are available to the clinician but the relative emphasis given to each is determined on an individual client basis. As only 13 participants had an early age at onset ( $<18$ years), it is unclear how effective recovery-focused CBT is likely to be for this group.

\section{Future research}

A larger-scale trial is needed to explore the cost-effectiveness of recovery-focused CBT and to provide more reliable estimates of the clinical benefits reported in the current study. It would also be of interest to evaluate the extent to which this approach can be adapted for individuals with an established course of bipolar disorder. Although current CBT approaches have reported mixed results with established bipolar disorder, it remains to be seen whether the more personalised approach of recovery-focused CBT has benefits for this group. As indicated by the current study, outcome measurement for therapy studies in bipolar disorder need to consider more carefully how to evaluate personal recovery and functioning outcomes to complement evaluations of episode recurrence.

Steven H. Jones, MSC (Clin Psychol), PhD, Spectrum Centre for Mental Health Research, Faculty of Health and Medicine, Lancaster University, Lancaster Gina Smith, PGDipPsych, DClinPsych, 5 Boroughs Partnership NHS Foundation Trus Warrington; Lee D. Mulligan, MSC, Manchester Mental Health and Social Care Trust, Manchester; Fiona Lobban, DClinPsy, PhD, Lancaster University, Lancaster; Heather Law, PhD, Psychosis Research Unit, Greater Manchester West NHS Foundation Trust, Manchester; Graham Dunn, MA, MSc, PhD, Institute of Population Health, University of Manchester; Mary Welford, DClinPsy, Psychosis Research Unit, Greater Manchester West NHS Foundation Trust; James Kelly, MSc, ClinPsyD, Lancashire Care NHS Foundation Trust, Lancaster; John Mulligan, MSC, ClinPsyD, The Beaco Service HMP Garth, Mersey Care NHS Trust, Liverpool; Anthony P. Morrison, ClinPsyD, Department of Clinical Psychology, University of Manchester Manchester, UK

Correspondence: Steven H. Jones, Spectrum Centre for Mental Health Research, Faculty of Health and Medicine, Lancaster University, Lancaster LA1 4YT, UK. Email: s.jones7@lancaster.ac.uk

First received 1 Nov 2013, final revision 16 Jun 2014, accepted 27 Jun 2014

\section{Funding}

National Institute for Health Research, England. This report presents independent research commissioned by the National Institute for Health Research (NIHR) under its Programm Grants for Applied Research funding scheme (RP-PG-0606-1086). Further support was received from primary care trusts, mental health trusts, the Mental Health Research Network and Comprehensive Local Research Networks in North West England. The views expressed in this publication are those of the authors and not necessarily those of the NHS, the NIHR or the Department of Health.

\section{Acknowledgements}

Acknowledgements of individual members of the Service User Reference Group, Yvonne Awenat, Rory Byrne, Ellen Hodson, Sam Omar, Liz Pitt, Jason Price, Tim Rawcliffe and Yvonne Thomas for their work on this study.

\section{References}

1 Barnard PJ. Bridging between basic theory and clinical practice. Behav Res Ther 2004; 42: 977-1000.

2 Jones S. Circadian rhythms, multilevel models of emotion and bipolar disorder - an initial step towards integration? Clin Psychol Rev 2001; 21 1193-209.
3 Post RM. Transduction of psychosocial stress into the neurobiology of recurrent affective disorder. Am J Psychiatry 1992; 149: 999-1010.

4 Scott J, Paykel E, Morriss R, Bentall R, Kinderman P, Johnson T, et al. Cognitive-behavioural therapy for severe and recurrent bipolar disorders: randomised controlled trial. Br J Psychiatry 2006; 188: 313-20.

5 Lish JD, Dime-Meenan S, Whybrow PC, Price RA, Hirschfeld RM. The national depressive and manic-depressive association (DMDA) survey of bipolar members. J Affect Disord 1994; 31: 281-94.

6 Baldessarini RJ, Tondo L, Vazquez GH, Undurraga J, Bolzani L, Yildiz A, et al. Age at onset versus family history and clinical outcomes in 1,665 international bipolar-I disorder patients. World Psychiatry 2012; 11: 40-6.

7 Mead S, Copeland ME. What recovery means to us. Comm Ment Health J 2000; 36: 315-28.

8 Department of Health: New Horizons: A Shared Vision for Mental Health. Department of Health, 2009.

9 Department of Health: No Health Without Mental Health: A CrossGovernment Mental Health Outcomes Strategy. Department of Health, 2011.

10 Jones SH, Mulligan LD, Law H, Dunn G, Welford M, Smith G, et al. A randomised controlled trial of recovery focused CBT for individuals with early bipolar disorder. BMC Psychiatry 2012; 12: 204.

11 Hamilton M. A rating scale for depression. I Neurol Psychiatry 1960; 23: 59-62.

12 Bech $\mathrm{P}$, Rafaelsen OJ, Kramp P, Bolwig TG. The mania rating scale: scale construction and inter-observer agreement. Neuropharmacology 1978; 17: 430-1.

13 Goldberg JF, Perlis RH, Bowden CL, Thase ME, Miklowitz DJ, Marangell LB, et al. Manic symptoms during depressive episodes in 1,380 patients with bipolar disorder: findings from the STEP-BD. Am J Psychiatry 2009; 166: $173-81$.

14 Perlis RH, Ostacher MJ, Patel JK, Marangell LB, Zhang $\mathrm{H}$, Wisniewski SR, et al. Predictors of recurrence in bipolar disorder: primary outcomes from the systematic treatment enhancement program for bipolar disorder (STEP-BD) Am J Psychiatry 2006; 163: 217-24.

15 American Psychiatric Association. Diagnostic and Statistical Manual of Mental Disorder (4th edn) (DSM-IV). APA, 1994.

16 First MB, Gibbon M, Spitzer RL, Williams JBW, Benjamin LS. Structured Clinical Interview for DSM-IV Axis I Disorders, Research Version, Patient Edition. American Psychiatric Press, 1997.

17 Keller MB, Lavori PW, Friedman B, Nielsen E, Endicott J, McDonald-Scott P, et al. The longitudinal interval follow-up evaluation. a comprehensive method for assessing outcome in prospective longitudinal studies. Arch Gen Psychiatry 1987; 44: 540-8.

18 Jones S, Mulligan LD, Higginson S, Dunn G, Morrison A. The bipolar recovery questionnaire: psychometric properties of a quantitative measure of recovery experiences in bipolar disorder. J Affect Disord, 2013; 147: 34-43.

19 Michalak EE, Murray G, Crest BD. Development of the QoL.BD: a disorderspecific scale to assess quality of life in bipolar disorder. Bipolar Disord 2010; 12: $727-40$

20 Morosini P, Magliano L, Brambilla L, Ugolini S, Pioli R. Development, reliability and acceptability of a new version of the DSMIV social and occupational functioning assessment scale (SOFAS) to assess routine social funtioning. Acta Psychiatr Scand 2000; 101: 323-9.

21 Beck AT. Cognitive models of depression. J Cogn Psychother 1987; 1: 5-37.

22 Bauer MS, Crits-Christoph P, Ball WA, Dewees E, McAllister T, Alahi P, et al. Independent assessment of manic and depressive symptoms by self-rating. Scale characteristics and implications for the study of mania. Arch Gen Psychiatry 1991; 48: 807-12.

23 Stephenson BJ, Rowe BH, Haynes RB, Macharia WM, Leon G. The rational clinical examination. Is this patient taking the treatment as prescribed? JAMA 1993; 269: 2779-81.

24 Tracey TJ, Kokotovic AM. Factor structure of the working alliance inventory. Psychol Assess 1989; 1: 207-10.

25 Blackburn IM, James IA, Milne DL, Baker C, Standart S, Garland A, Reichelt FK. The revised cognitive therapy scale (CTS-R): psychometric properties. Behav Cogn Psychother 2001; 29: 431-46.

26 Miklowitz D, Goodwin GM, Bauer MS, Geddes JR. Common and specific elements of psychosocial treatments for bipolar disorder: a survey of clinicians participating in randomized trials. J Psychiatr Pract 2008; 14 77-85.

27 Jones S, Burrell-Hodgson G. Cognitive behavioural treatment of first diagnosis bipolar disorder. Clin Psychol Psychother 2008; 15: 367-77.

28 Dunn G, Maracy M, Dowrick C, Ayuso-Mateos JL, Dalgard OS, Page H, et al. Estimating psychological treatment effects from a randomised controlled trial 
with both non-compliance and loss to follow-up. Br J Psychiatry 2003; 183 323-31.

29 Loeys T, Goetghebeur E. A causal proportional hazards estimator for the effect of treatment actually received in a randomized trial with all-or-nothing compliance. Biometrics 2003; 59: 100-5.

30 Frangakis CE, Rubin DB. Addressing complications of intention-to-treat analysis in the combined presence of all-or-none treatment-noncompliance and subsequent missing outcomes. Biometrika 1999; 86: 365-79.

31 Busseri MA, Tyler, JD. Interchangeability of the Working Alliance Inventory and Working Alliance Inventory, Short Form. Psychol Assess 2003; 15: 193-7.

32 Davidson K, Norrie J, Tyrer P, Gumley A, Tata P, Murray H, et al. The effectiveness of cognitive behavior therapy for borderline personality disorder: Results from the Borderline Personality Disorder Study of Cognitive Therapy (BOSCOT) trial. J Pers Disord 2006; 20: 450-65.

33 Keen AJ, Freeston $\mathrm{MH}$. Assessing competence in cognitive-behavioural therapy. Br J Psychiatry 2008; 193: 60-4.

34 Mental Health Research Network. Service User Involvement in the UK Mental Health Research Network. MHRN, 2005.
35 Lam DH, Watkins ER, Hayward P, Bright J, Wright K, Kerr N, et al. A randomized controlled study of cognitive therapy for relapse prevention for bipolar affective disorder. Arch Gen Psychiatry 2003; 60: 145-52.

36 Fowler D, Hodgekins J, Painter M, Reilly T, Crane C, MacMillan I, et al. Cognitive behaviour therapy for improving social recovery in psychosis: a report for the ISREP MRC trial platform study (Improving social recovery in early psychosis). Psychol Med 2009; 39: 1627-36.

37 Ball JR, Mitchell PB, Corry JC, Skillecorn A, Smith M, Malhi GS. A randomized controlled trial of cognitive therapy for bipolar disorder: Focus of long-term change. J Clin Psychiatry 2006; 67: 277-86.

38 Meyer TD, Hautzinger, M. Cognitive behaviour therapy and supportive therapy for bipolar disorders: relapse rates for treatment period and 2-year follow-up. Psychol Med 2012; 42: 1429-39.

39 Zaretsky A, Lancee W, Miller C, Harris A, Parikh SV. Is cognitive-behavioural therapy more effective than psychoeducation in bipolar disorder? Can J Psychiatry 2008; 53: 441-88.

40 Lobban F, Taylor L, Chandler E, Tyler E, Kinderman P, Kolamunnage-Donna R, et al. Enhanced relapse prevention for bipolar disorder by community mental health teams: cluster feasibility randomised trial. Br J Psychiatry 2010; 196: 59-63.

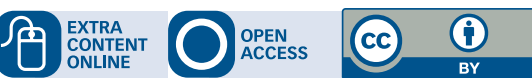

